\title{
Oral Plasma Kallikrein Inhibitor for Prophylaxis in Hereditary Angioedema
}

\author{
E. Aygören-Pürsün, A. Bygum, V. Grivcheva-Panovska, M. Magerl, J. Graff, \\ U.C. Steiner, O. Fain, A. Huissoon, T. Kinaciyan, H. Farkas, R. Lleonart, \\ H.J. Longhurst, W. Rae, M. Triggiani, W. Aberer, M. Cancian, A. Zanichelli, \\ W.B. Smith, M.L. Baeza, A. Du-Thanh, M. Gompels, T. Gonzalez-Quevedo, \\ J. Greve, M. Guilarte, C. Katelaris, S. Dobo, M. Cornpropst, D. Clemons, \\ L. Fang, P. Collis, W. Sheridan, M. Maurer, and M. Cicardi
}

The authors' full names, academic degrees, and affiliations are listed in the Appendix. Address reprint requests to Dr. Aygören-Pürsün at the Department of Children and Adolescents, University Hospital Frankfurt, Goethe University, TheodorStern-Kai 7, D-60590 Frankfurt, Germany, or at aygoeren@em.uni-frankfurt.de.

Drs. Aygören-Pürsün, Bygum, Maurer, and Cicardi contributed equally to this article.

N Engl J Med 2018;379:352-62. DOI: 10.1056/NEJMoal716995 Copyright @ 2018 Massachusetts Medical Society.
ABSTRACT

\section{BACKGROUND}

Hereditary angioedema is a life-threatening illness caused by mutations in the gene encoding $\mathrm{C} 1$ inhibitor (also called $\mathrm{C} 1$ esterase inhibitor) that lead to overactivation of the kallikrein-bradykinin cascade. BCX7353 is a potent oral smallmolecule inhibitor of plasma kallikrein with a pharmacokinetic and pharmacodynamic profile that may help prevent angioedema attacks.

\section{METHODS}

In this international, three-part, dose-ranging, placebo-controlled trial, we evaluated four doses of BCX7353 (62.5 mg, $125 \mathrm{mg}, 250 \mathrm{mg}$, and $350 \mathrm{mg}$ once daily) for the prevention of angioedema attacks over a 28-day period. Patients with type I or II hereditary angioedema with a history of at least two angioedema attacks per month were randomly assigned to BCX7353 or placebo. The primary efficacy end point was the number of confirmed angioedema attacks. Key secondary end points included angioedema attacks according to anatomical location and quality of life.

\section{RESULTS}

A total of 77 patients underwent randomization, 75 received BCX7353 or placebo, and 72 completed the trial. The rate of confirmed angioedema attacks was significantly lower among patients who received BCX7353 at daily doses of $125 \mathrm{mg}$ or more than among those who received placebo, with a $73.8 \%$ difference at $125 \mathrm{mg}$ $(\mathrm{P}<0.001)$. Significant benefits with respect to quality-of-life scores were observed in the 125 -mg and $250-\mathrm{mg}$ dose groups $(\mathrm{P}<0.05)$. Gastrointestinal adverse events, predominantly of grade 1 , were the most commonly reported adverse events, particularly in the two highest BCX7353 dose groups.

\section{CONCLUSIONS}

Once-daily oral administration of BCX7353 at a dose of $125 \mathrm{mg}$ or more resulted in a significantly lower rate of attacks of hereditary angioedema than placebo. Mild gastrointestinal symptoms were the principal side effect. (Funded by BioCryst Pharmaceuticals; APeX-1 ClinicalTrials.gov number, NCT02870972.) 
$\mathrm{H}$ EREDITARY ANGIOEDEMA IS A GENETIC disorder characterized by recurrent episodes of swelling. ${ }^{1,2}$ Oropharyngeal swelling can be life-threatening ${ }^{3}$, and attacks in other sites, including the limbs, genitalia, face, and intestines, can be painful, disabling, and disfiguring and have a substantial effect on function and quality of life. ${ }^{4}$ Most patients with hereditary angioedema have mutations in the regulatory or coding regions of the gene that encodes $\mathrm{C} 1$ inhibitor (SERPING1), which result in either a deficiency (type I) or dysfunction (type II) of C1 inhibitor. $^{5}$

$\mathrm{C} 1$ inhibitor (also called C1 esterase inhibitor) is a serine protease inhibitor that normally prevents uncontrolled contact activation and bradykinin production by covalently binding to and inactivating plasma kallikrein. ${ }^{6}$ Increased bradykinin generation leads to increased vascular permeability and swelling.

Plasma-derived purified C1 inhibitor is approved for the prophylactic treatment of hereditary angioedema through intravenous or subcutaneous injection. The only available effective drugs for oral prophylaxis against angioedema attacks are attenuated androgens, such as danazol, and tranexamic acid. Although administration of androgens is convenient, unacceptable adverse effects (e.g., virilizing androgenic hormonal effects, hypertension, and an increased risk of hepatocellular adenoma or carcinoma) and several contraindications (e.g., in growing children and pregnant women) limit their clinical use. ${ }^{7-9}$

BCX7353 is a potent, synthetic small molecule that inhibited plasma kallikrein with once-daily oral administration in healthy volunteers. ${ }^{10}$ In contrast to parenterally administered options under development or commercially available for prophylaxis against angioedema attacks, inhibition of kallikrein with an orally bioavailable small molecule such as BCX7353 offers the distinct advantage of oral administration.

\section{METHODS}

TRIAL DESIGN

The Angioedema Prophylaxis 1 (APeX-1) trial was a phase 2, randomized, double-blind, pla- cebo-controlled, parallel-group, three-part, doseresponse trial that evaluated the safety, adverseevent profile, pharmacokinetics, pharmacodynamics,and efficacy of BCX7353 in patients with hereditary angioedema and C1 inhibitor deficiency. The trial was designed to establish proof of concept that a high dose of BCX7353, administered once daily for 28 days, was safe and effective in reducing the rate of angioedema attacks. Once proof of concept was established, additional doses were evaluated to characterize the dose response. On the basis of pharmacokinetic and pharmacodynamic modeling from a completed phase 1 study, the protocol was initially designed as a two-part trial with oncedaily doses of $350 \mathrm{mg}, 250 \mathrm{mg}$, and $125 \mathrm{mg}$ of BCX7353. After the trial was initiated, the protocol was amended to include a lower dose of $62.5 \mathrm{mg}$ of BCX7353 once daily in part 3. Eligible patients underwent central randomization to BCX7353 or placebo within each part of the trial. Patients participated in one trial part only. Further details of the trial design and randomization are provided in the protocol (available with the full text of this article at NEJM.org) and in Section S2 in the Supplementary Appendix (available at NEJM.org).

The trial was designed by the sponsor (BioCryst Pharmaceuticals) in collaboration with the first, next-to-last, and last authors. The protocol was approved by regulatory authorities and independent ethics committees. All the patients provided written informed consent before the conduct of any trial-related procedures.

Investigators and site personnel collected data in collaboration with the sponsor, and the sponsor and PharStat (a contract research organization paid by the sponsor) analyzed the data. All the authors had access to the data (nondisclosure agreements were in place). The first, next-to-last, and last authors contributed to the analysis and interpretation of the data. All the authors reviewed and approved the manuscript for publication and vouch for the completeness and accuracy of the data and analyses and for the adherence of the trial to the protocol. Technical support in the drafting of the manuscript, including preparation of the first draft, was provided by J Anderson Solutions, funded by the sponsor. 


\section{PATIENTS AND PATIENT RECRUITMENT}

Eligible male or female patients were 18 to 70 years of age with a clinical diagnosis of type I or type II hereditary angioedema. Patients were required to have a documented rate of angioedema attacks of at least two attacks per month for 3 consecutive months within the 6 months before the screening visit. Patients were excluded if they were suspected of having C1 inhibitor resistance (i.e., they were having regular attacks despite C1 inhibitor treatment for routine prophylaxis) or if they were using a $\mathrm{C} 1$ inhibitor, androgens, or tranexamic acid for prophylaxis of attacks within 7 days before screening. Treatment of acute attacks, including the use of a $\mathrm{C} 1$ inhibitor, was not excluded. Complete inclusion and exclusion criteria are provided in Table S3 in the Supplementary Appendix.

\section{TRIAL REGIMENS}

Blinded trial medication with matching placebo was supplied as capsules for daily oral administration for 28 days. Patients were permitted to use their usual medication (including icatibant and intravenous and subcutaneous C1 inhibitor) to treat any attacks that occurred at any time during the trial.

\section{CLINICAL ASSESSMENTS}

Patients were provided with paper diaries for daily recording of trial-regimen administration and angioedema attacks. If patients had an attack, they were to provide details of the symptoms, severity (using the Angioedema Activity Score), ${ }^{11}$ triggers, and duration of the attack and medications administered to treat the attack. To assess the effect of the disease on the patient's quality of life, the Angioedema Quality of Life Questionnaire $(\mathrm{AE}-\mathrm{QoL})^{12}$ was administered at the start and end of the intervention phase. Patients returned to the clinic for routine monitoring of vital signs, 12-lead electrocardiography, pulmonary diffusion testing, physical examinations, laboratory assessments of serum biochemical and hematologic values, liver-enzyme testing, urinalysis, and documentation of adverse events. Blood samples for pharmacokinetic and pharmacodynamic testing were obtained from patients before dose administration (on days 1, 14, and 29) and for up to 24 hours after the dose on day 14 . Blood samples for measurement of functional levels of $\mathrm{C} 1$ inhibitor were obtained on days 1 and 29.

\section{EFFICACY}

The primary end point of the trial was the number of confirmed angioedema attacks during the effective dosing period (days 8 to 28 , inclusive). All patient-reported attacks were adjudicated by an independent clinical end-point adjudication panel whose members were unaware of the trialgroup assignments. Panel membership and details regarding the adjudication process are provided in Section S1 in the Supplementary Appendix. Attacks that were confirmed by the adjudication panel were included in efficacy analyses.

Key secondary end points of the trial included the number of angioedema attacks categorized according to anatomical location and the change from baseline in scores on the disease-specific and validated AE-QoL. The AE-QoL measures quality of life across four domains (functioning, fatigue and mood, fears and shame, and food) and derives a total score on a scale from 0 to 100, with higher scores indicating greater impairment. A minimal clinically important difference of 6 points has been described..$^{13}$ Other secondary efficacy end points are presented in Table S7 in the Supplementary Appendix.

\section{SAFETY}

The safety and adverse-event profile of BCX7353 were assessed by means of laboratory testing and monitoring of patients for adverse events that occurred from the time of the first dose to the time of the last dose plus 30 days. During the conduct of the trial, an independent data monitoring committee (Table S2 in the Supplementary Appendix) provided safety oversight by reviewing blinded data and advising the sponsor on safety-related findings.

\section{PHARMACOKINETICS AND PHARMACODYNAMICS}

Steady-state pharmacokinetic properties of BCX7353 - the maximum plasma concentration $\left(\mathrm{C}_{\max }\right)$, the observed trough plasma concentration at the end of the dosing interval $\left(\mathrm{C}_{\text {tau }}\right)$, the time at which $\mathrm{C}_{\max }$ occurred, and the area under the plasma concentration-time curve during the dosing interval $\left(\mathrm{AUC}_{\mathrm{tau}}\right)$ - were additional secondary end points, as was the ex vivo 
kallikrein inhibition activity of BCX7353 (analyzed as described previously ${ }^{10}$ ).

\section{STATISTICAL ANALYSIS}

In part 1 of the trial, we calculated that a sample of 18 patients per group would provide a power of $93 \%$ to detect a difference in the attack rate of 0.5 attacks per week between the 350-mg BCX7353 group and the placebo group at an alpha level of 0.05 , assuming a mean baseline rate of 1 attack per week. The primary efficacy end point of the trial was the number of confirmed angioedema attacks in the intention-to-treat and per-protocol populations. Population definitions and numbers of patients included in each population are summarized in Section S4 in the Supplementary Appendix. The number of angioedema attacks was analyzed according to trial group with the use of appropriate descriptive statistics for the weekly attack rate, change and percentage change from baseline in attack rate, proportion of patients with no attacks, and number and percent of attack-free days. Efficacy analyses were conducted for angioedema attacks reported during the entire dosing interval (days 1 to 28 , inclusive) and during the effective dosing period in which BCX7353 levels were at a steady state (days 8 to 28 , inclusive).

Between-group comparisons (BCX7353 vs. placebo) for the weekly attack rate were performed with the use of an analysis-of-covariance model. The adjusted qualifying attack rate was included as a covariate in the model. The least-squares mean for each trial group and the relative difference and associated 95\% confidence intervals in attack rate between the placebo group and each BCX7353 dose group were calculated.

Data for the primary end point are shown for the effective dosing period in the intention-totreat population, since this would best assess the effectiveness of the trial medication after steady-state levels had been achieved in this small, phase 2 trial. Data are also presented for the per-protocol population and during the entire dosing period. The primary efficacy analysis was conducted without imputation for missing data (Tables S5 and S6 in the Supplementary Appendix).

Analyses of the primary end point of the number of confirmed attacks, followed by the secondary end point of AE-QoL scores, were per- formed with the use of post hoc hierarchical testing to preserve the prespecified level of significance of 0.05 . Safety analyses were based on the safety population and included all randomly assigned patients who received a dose of a trial regimen. All statistical analyses were performed with the use of SAS software (version 9.3).

\section{RESULTS}

\section{PATIENTS}

The trial was initiated in August 2016, and the last patient observation occurred in August 2017. A total of 86 patients were screened from 26 sites across Europe, Canada, and Australia. Of the 77 patients who underwent randomization, 2 never received a trial regimen or entered any diary data and 3 discontinued the trial regimen owing to an adverse event or laboratory abnormality. Details regarding disposition of the patients and discontinuation are presented in Figure S2 and Table S4, respectively, in the Supplementary Appendix.

The trial groups were generally balanced with respect to age and qualifying attack rate, although the proportion of women was not evenly distributed across groups, and patients in the 350-mg dose group had a higher rate of previous androgen use than the other groups (Table 1). Baseline C1 inhibitor levels were low, with median values ranging from $19 \%$ to $34 \%$ of normal values. Sequential enrollment into parts 1, 2, and 3 may have contributed to the differences in demographic and clinical characteristics that were observed among the trial groups.

\section{EFFICACY RESULTS}

Primary End Point

The primary efficacy end point was the number of confirmed angioedema attacks. Significant and clinically meaningful differences in the rate of attacks were observed between patients who received BCX7353 at doses of $125 \mathrm{mg}$ or more once daily and those who received placebo (Table 2). The least-squares mean rate of attacks among patients who received placebo (pooled across all three parts of the trial) was 0.95 per week, and the least-squares mean percent differences in the confirmed rate of attacks per week according to $\mathrm{BCX} 7353$ dose group were as follows: $350 \mathrm{mg},-45.5 \%(\mathrm{P}=0.006) ; 250 \mathrm{mg},-44.6 \%$ 


\begin{tabular}{|c|c|c|c|c|c|}
\hline \multirow[t]{2}{*}{ Characteristic } & \multicolumn{4}{|c|}{ BCX7353 } & \multirow[t]{2}{*}{$\begin{array}{l}\text { Placebo } \\
(\mathrm{N}=23)\end{array}$} \\
\hline & $\begin{array}{l}62.5 \mathrm{mg} \\
(\mathrm{N}=7)\end{array}$ & $\begin{array}{l}125 \mathrm{mg} \\
(\mathrm{N}=14)\end{array}$ & $\begin{array}{l}250 \mathrm{mg} \\
(\mathrm{N}=15)\end{array}$ & $\begin{array}{l}350 \mathrm{mg} \\
(\mathrm{N}=18)\end{array}$ & \\
\hline Age $-y r$ & $38.9 \pm 16.6$ & $48.1 \pm 12.6$ & $39.5 \pm 13.9$ & $43.8 \pm 11.6$ & $46.6 \pm 10.9$ \\
\hline Female sex — no. (\%) & $6(86)$ & $10(71)$ & $7(47)$ & $11(61)$ & $13(57)$ \\
\hline \multicolumn{6}{|l|}{ Race-no. (\%)† } \\
\hline White & $7(100)$ & $14(100)$ & $12(80)$ & $15(83)$ & $21(91)$ \\
\hline Asian & 0 & 0 & $1(7)$ & $1(6)$ & 0 \\
\hline Other & 0 & 0 & $2(13)$ & $2(11)$ & $2(9)$ \\
\hline Previous androgen use — no. (\%) & $3(43)$ & $4(29)$ & $8(53)$ & $15(83)$ & $13(57)$ \\
\hline Angioedema attacks/wk & $1.15 \pm 0.52$ & $0.99 \pm 0.52$ & $0.93 \pm 0.45$ & $0.90 \pm 0.44$ & $1.03 \pm 0.57$ \\
\hline $\begin{array}{l}\text { Median } \mathrm{Cl} \text { inhibitor function (IQR) - \% } \\
\text { of normal value }\end{array}$ & $11(7-47)$ & $15(11-35)$ & $15(6-28)$ & $19(5-28)$ & $10(4-37)$ \\
\hline Alanine aminotransferase $-U /$ liter & $18.4 \pm 6.6$ & $22.0 \pm 9.5$ & $31.0 \pm 20.5$ & $42.5 \pm 44.1$ & $29.0 \pm 13.0$ \\
\hline \multicolumn{6}{|c|}{$\begin{array}{l}\text { Plus-minus values are means } \pm \text { SD. The intention-to-treat population included all the patients who underwent random- } \\
\text { ization. IQR denotes interquartile range. } \\
\text { Race was reported by the patient. } \\
\text { The baseline attack rate was based on patient-reported attacks over a 3-month period. }\end{array}$} \\
\hline
\end{tabular}

$(\mathrm{P}=0.01) ; 125 \mathrm{mg},-73.8 \%(\mathrm{P}<0.001)$; and $62.5 \mathrm{mg}$, $-10.5 \%(P=0.64)$.

When attack rates were analyzed according to anatomical location, a differential response was observed for peripheral attacks as compared with abdominal attacks, particularly at the 250-mg and 350-mg doses of BCX7353 (Table 2). Although the rate of peripheral attacks was lower with BCX7353 than with placebo at all doses of $125 \mathrm{mg}$ or more, the rate of abdominal attacks was lower with BCX7353 than with placebo at the 125-mg dose only.

\section{Secondary End Points}

The percent of attack-free days and the proportion of patients who were attack-free were higher with BCX7353 at doses of $125 \mathrm{mg}$ or more than with placebo (Table 2). An important secondary end point in the trial was quality of life as measured by the AE-QoL. The least-squares mean change from baseline in the AE-QoL total score was -29.0 in the 125-mg dose group, as compared with -4.5 in the placebo group (difference, -24.5; $\mathrm{P}<0.001$ ), indicating a significant benefit with the 125-mg dose. There were also significant differences favoring the 125-mg dose across three of the four domains of the AE-QoL ( $\mathrm{P} \leq 0.006$ vs. placebo) (Fig. 1). A significant difference between the 250-mg dose group and the placebo group was observed for the functioning domain $(\mathrm{P}=0.02)$, in favor of the 250-mg dose. Results for other secondary end points, including the rate of attacks resulting in treatment and the overall severity of illness, support the efficacy of BCX7353 at doses of $125 \mathrm{mg}$ or more (Table S7 in the Supplementary Appendix). None of the patients discontinued the trial medication owing to lack of efficacy.

\section{PHARMACOKINETICS AND PHARMACODYNAMICS}

After administration of multiple daily doses of

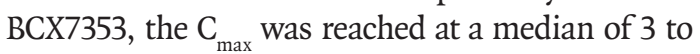
4 hours after dosing (Fig. 2A). At doses of $125 \mathrm{mg}$ or more, geometric mean plasma trough concentrations $\left(\mathrm{C}_{\mathrm{tau}}\right)$ were generally maintained above the minimum target concentration range (approximately 4 times the half-maximal effective concentration $\left.\left[\mathrm{EC}_{50}\right]\right)$ throughout the dosing interval. There was a greater-than-dose-proportional increase in exposure $\left(\mathrm{AUC}_{\mathrm{tau}}\right.$ and $\mathrm{C}_{\max }$ ) across the dose range of $62.5 \mathrm{mg}$ to $350 \mathrm{mg}$, with an increase in exposure of approximately 11 times with an increase in dose of 4.6 times (Table S8 in the Supplementary Appendix). A dose-dependent inhibition of kallikrein was observed with BCX7353 treatment across the dose range (Fig. 2B), 


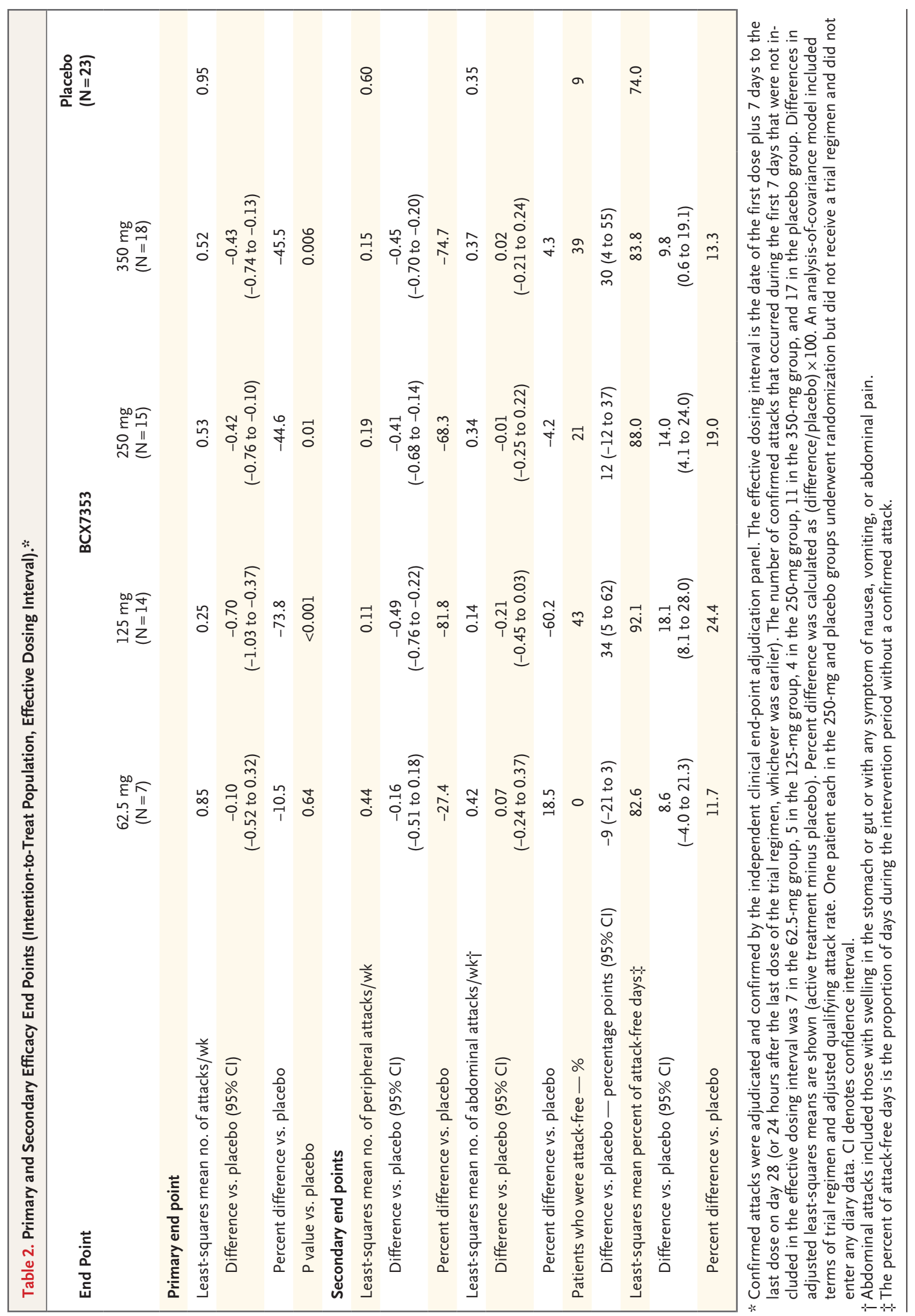




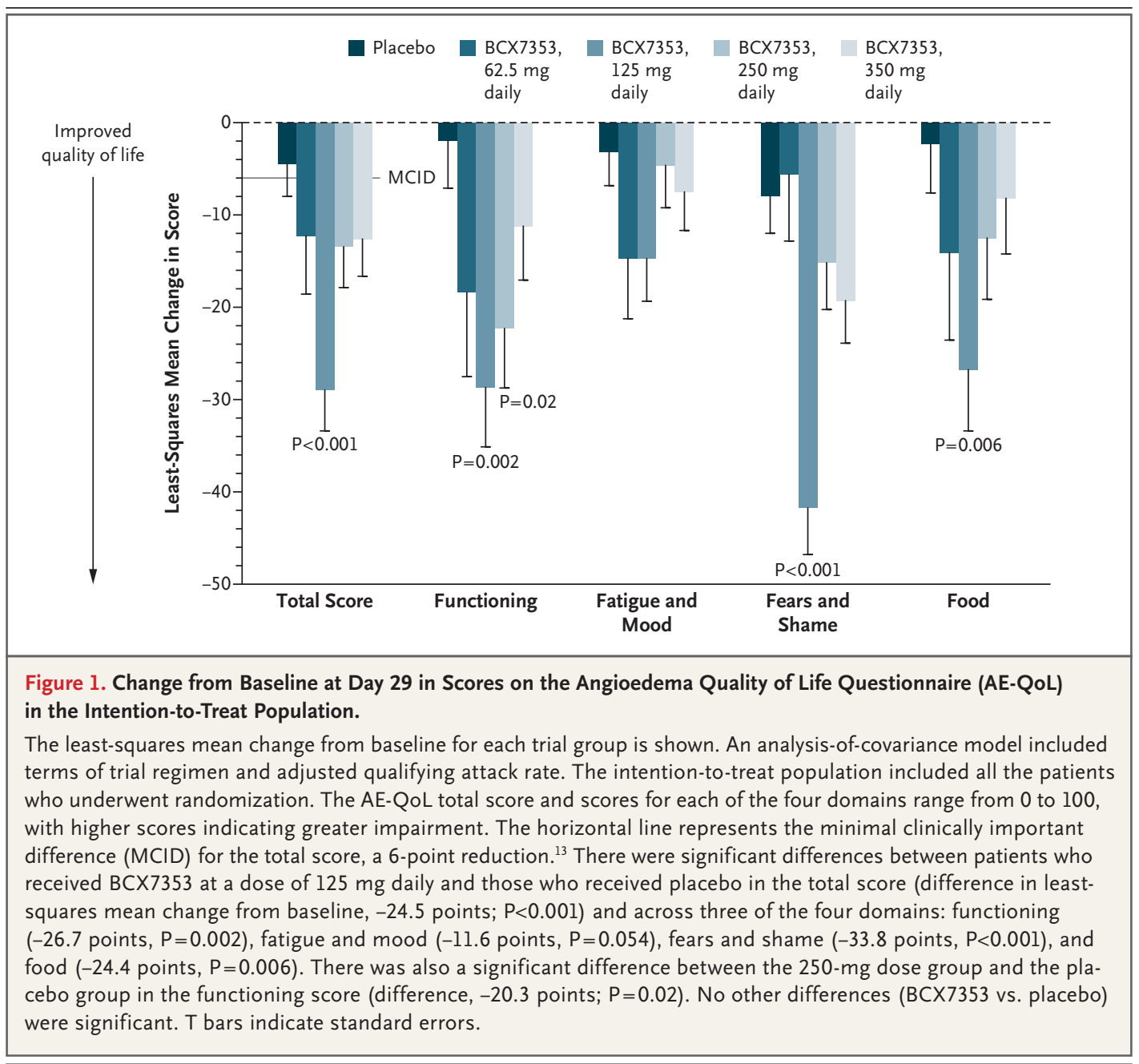

a finding consistent with the exposure data. The drug effect on kallikrein inhibition was correlated with exposure $\left(r^{2}=0.75\right)$ (data not shown).

\section{SAFETY}

Three patients who received BCX7353 at a dose of $350 \mathrm{mg}$ discontinued the trial regimen owing to adverse events (Table 3). A total of $67 \%$ of all adverse events were mild in severity (grade 1). Analysis of the adverse-event profile identified a dose-related increase in the incidence of gastrointestinal events. Gastrointestinal events were most commonly reported in the 350-mg dose group (44\%) and 250-mg dose group (50\%), with considerably fewer in the $125 \mathrm{-mg}$ dose group (29\%) and 62.5-mg dose group (14\%); the incidence in the 62.5-mg dose group was similar to that in the placebo group (18\%). Gastrointestinal events were generally mild (grade $1,75 \%$ of treated patients with such events) to moderate (grade 2, 20\%) in severity, although one patient in the 350-mg dose group had severe (grade 3) abdominal pain that led to discontinuation of the trial regimen (Table 3). Only mild (grade 1) gastrointestinal events occurred in the 125-mg and 62.5-mg dose groups.

A total of three patients who received BCX7353 had mild (grade 1) or moderate (grade 2) liverrelated adverse events (one patient in the $250-\mathrm{mg}$ dose group and one in the 350 -mg dose group had grade 1 events, and one patient in the 350-mg dose group had a grade 2 event). Two additional patients in the 350-mg dose group had a grade 3 or grade 4 liver-enzyme abnormality: one patient had a grade 3 elevation in the $\gamma$-glutamyltransferase level (3.4 times the upper limit of the normal range), and the other patient had a grade 3 elevation in the alanine aminotransferase level (3.9 times the upper limit of the normal range) and a grade 4 elevation in the $\gamma$-glutamyltransferase 
Figure 2. Dose of BCX7353, Concentration-Time Profile at Steady State, and Plasma Kallikrein Inhibition.

On day 14, serial blood samples were drawn for pharmacokinetic analysis (Panel A) before dose administration and at the following time points: $1,2,3,4,5,6,8$, and 24 hours after dose administration. For the 24 -hour postdose samples, patients returned to the clinic and had the blood sample drawn before dose administration on day 15 . On day 29 , a trough pharmacokinetic blood sample was drawn. However, if serial pharmacokinetic samples could not be obtained on day 14 , steady-state serial pharmacokinetic samples were obtained on day 28 , with a 24-hour pharmacokinetic sample drawn at the scheduled visit on day 29. The plasma samples for determination of BCX7353 concentrations were analyzed with the use of a validated liquid chromatography-mass spectrometry assay. In this trial, none of the patients who received $62.5 \mathrm{mg}$ of $\mathrm{BCX} 7353$ met or exceeded the target of 4 times the $\mathrm{EC}_{50}$ across the 24-hour dosing interval, whereas 9 of 14 patients who received $125 \mathrm{mg}$ met or exceeded the target, and all the patients who received $250 \mathrm{mg}$ or $350 \mathrm{mg}$ exceeded the target concentration. Kallikrein inhibition (Panel B) was measured in an ex vivo fluorogenic assay. ${ }^{10}$ The assay uses ellagic acid to activate the contact pathway and convert prekallikrein to kallikrein. A fluorogenic substrate that is specific for the enzyme ( $\mathrm{N}$-carbobenzyloxy-phenylalanine-arginine7-amino-4-methylcoumarin) was used to measure its amidolytic activity. Owing to the dilution in the sample preparation for the assay, the BCX7353 concentration in the ex vivo assay represents one quarter of the corresponding in vivo plasma concentration. The results of kallikrein activity were expressed as percent inhibition as compared with the activity present in the predose plasma sample on day 1 . At doses of $250 \mathrm{mg}$ or $350 \mathrm{mg}$ of BCX7353, maximum kallikrein inhibition was approximately $90 \%$, and inhibition of more than $75 \%$ was maintained throughout the 24 -hour dosing period. Maximum kallikrein inhibition with 125 mg of BCX7353 was approximately $60 \%$, with $45 \%$ inhibition at 24 hours, whereas at a dose of $62.5 \mathrm{mg}$, maximum inhibition was approximately $30 \%$, with $8 \%$ kallikrein inhibition at 24 hours.

level (10.7 times the upper limit of the normal range). Both patients had elevated liver-enzyme levels at baseline.

All five of these patients had extensive histories of previous androgen use. These patients' laboratory values returned to normal or baseline without intervention once dosing was completed. No liver-related adverse events or grade 3 or 4 liver-enzyme abnormalities were observed at the 125-mg or 62.5-mg doses.

\section{DISCUSSION}

The APeX-1 trial showed that BCX7353 at doses of $125 \mathrm{mg}$ or more administered orally once daily resulted in markedly lower rates of angioedema

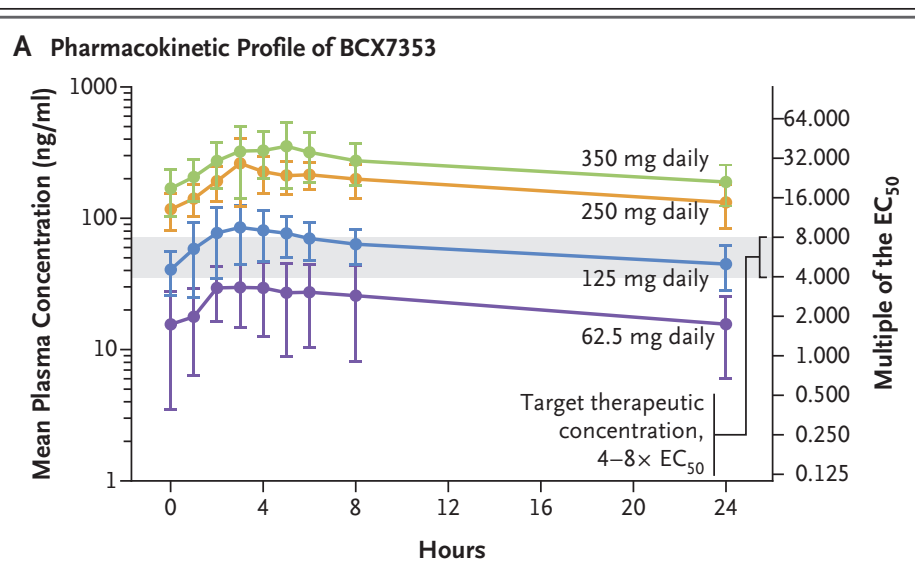

B Inhibition of Plasma Kallikrein

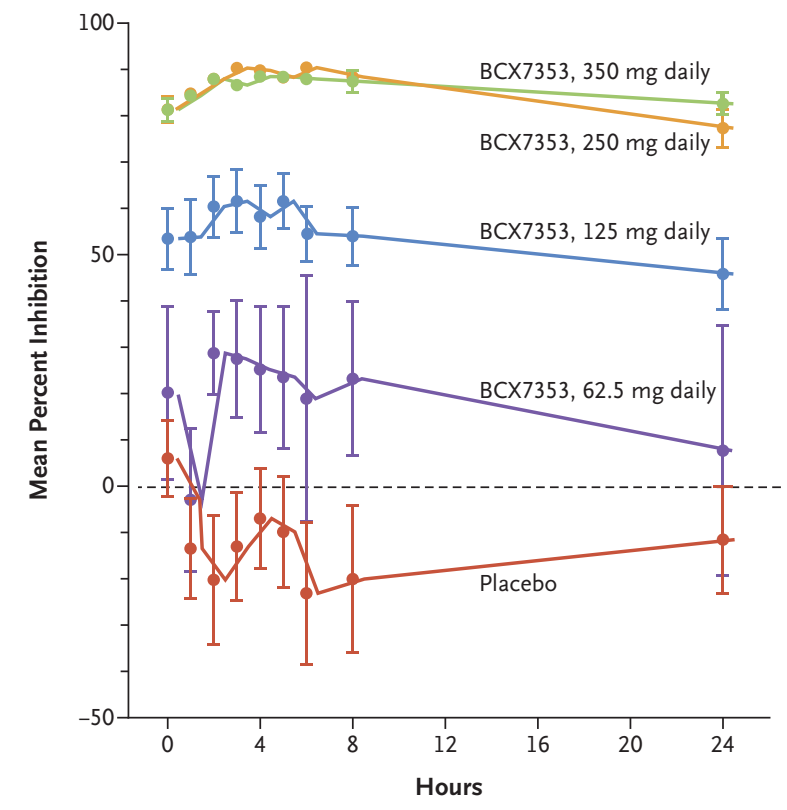

attacks than placebo. An apparent U-shaped dose response was observed in the primary end point, with the highest treatment effect observed at the 125-mg dose: the attack rate was $73.8 \%$ lower than with placebo, and $43 \%$ of patients were attack-free.

The data suggest that the efficacy of the BCX7353 doses of $250 \mathrm{mg}$ and $350 \mathrm{mg}$ was probably masked by gastrointestinal adverse events that may have been misattributed as early symptoms of abdominal angioedema attacks. Only the 125-mg dose group had a lower rate of abdominal attacks than the placebo group, whereas all groups that received BCX7353 at doses of $125 \mathrm{mg}$ or more had lower rates of peripheral attacks than the placebo group (difference, $68 \%$ 
Table 3. Summary of Adverse Events (Safety Population).*

Event

\begin{tabular}{|c|c|c|c|c|c|}
\hline & $\begin{array}{c}62.5 \mathrm{mg} \\
(\mathrm{N}=7)\end{array}$ & $\begin{array}{l}125 \mathrm{mg} \\
(\mathrm{N}=14)\end{array}$ & $\begin{array}{l}250 \mathrm{mg} \\
(\mathrm{N}=14)\end{array}$ & $\begin{array}{l}350 \mathrm{mg} \\
(\mathrm{N}=18)\end{array}$ & \\
\hline & \multicolumn{5}{|c|}{ number of patients (percent) } \\
\hline Any adverse event & $4(57)$ & $7(50)$ & $11(79)$ & $14(78)$ & $15(68)$ \\
\hline Any serious adverse event & 0 & 0 & $1(7) \dagger$ & 0 & 0 \\
\hline Grade 3 adverse event considered to be related to the trial regiment & 0 & 0 & 0 & $1(6)$ & 0 \\
\hline Adverse event leading to discontinuation of the trial regimen & 0 & 0 & 0 & $3(17) \mathbb{\int}$ & 0 \\
\hline \multicolumn{6}{|l|}{ Most commonly reported adverse events } \\
\hline Any gastrointestinal adverse event & $1(14)$ & $4(29)$ & $7(50)$ & $8(44)$ & $4(18)$ \\
\hline Abdominal pain $\|$ & $1(14)$ & $3(21)$ & $2(14)$ & $3(17)$ & 0 \\
\hline Diarrhea & 0 & 0 & $2(14)$ & $4(22)$ & $2(9)$ \\
\hline Flatulence & 0 & 0 & 0 & $2(11)$ & 0 \\
\hline Nausea & 0 & 0 & $3(21)$ & $3(17)$ & 0 \\
\hline Vomiting & 0 & 0 & 0 & $2(11)$ & 0 \\
\hline Fatigue & $1(14)$ & 0 & 0 & $2(11)$ & $1(5)$ \\
\hline Nasopharyngitis & $2(29)$ & 0 & $1(7)$ & $5(28)$ & $6(27)$ \\
\hline Liver-related event*** & 0 & 0 & $1(7)$ & $2(11)$ & 0 \\
\hline Headache & $2(29)$ & $2(14)$ & $1(7)$ & $1(6)$ & $4(18)$ \\
\hline
\end{tabular}

* The safety population included all randomly assigned patients who received a dose of a trial regimen. Shown are events that started after the date and time of the first dose to the last dose plus 30 days. Adverse events were graded according to the Division of Microbiology and Infectious Diseases Adult Toxicity Table (November 2007): grade 1 indicated mild, grade 2 moderate, grade 3 severe, and grade 4 lifethreatening. No patient had a grade 4 adverse event.

$\dagger$ A grade 2 gastrointestinal infection was considered to be unrelated to the trial regimen. Abdominal symptoms of gastrointestinal infection were similar to those in several previous (non-hereditary angioedema attack) episodes occurring during the previous 3 years that resulted in severe vomiting and diarrhea and an overnight hospitalization for intravenous fluids and antiemetics.

$¥$ In addition to the adverse events that were considered by the investigator to be related to the trial regimen, two patients in the 350-mg dose group had grade 3 adverse events that were considered to be unrelated to the trial regimen. One patient had a persistent grade 3 elevation in the alanine aminotransferase (ALT) level that was present at baseline, before the first dose of the trial regimen. One patient had a grade 3 gastrointestinal infection 1 week after completion of the last dose of the trial regimen.

$\int$ Reasons for discontinuation were grade 1 liver disorder (in one patient), grade 3 elevation in the ALT level (in one patient), and grade 3 abdominal pain and grade 2 vomiting (in one patient). The liver disorder was considered to be probably related to the trial regimen and occurred after a case of grade 1 gastroenteritis; the patient discontinued the trial regimen on day 18. Increases in levels of ALT (1.9 times the upper limit of the normal range [ULN] [grade 1]), $\gamma$-glutamyltransferase (5.4 times the ULN [grade 3]), and alkaline phosphatase (1.6 times the ULN [grade 1]) were observed, although levels of aspartate aminotransferase and bilirubin remained within the normal range. The grade 3 elevation in the ALT level was considered unlikely to be related to the trial regimen, and although the patient's ALT level was less than 2 times the ULN at screening (44 IU per liter, or 1.3 times the ULN [grade 1]) (i.e., the patient met the inclusion criteria), the patient entered the trial with an elevated ALT level (123 IU per liter, or 3.7 times the ULN [grade 3]) at baseline that remained at grade 2 or higher during the trial; the patient discontinued the trial regimen on day 23. The patient had received danazol ( $100 \mathrm{mg})$ before trial entry. The abdominal pain and vomiting were considered to be possibly related to the trial regimen and occurred two times before the third episode that resulted in discontinuation of the trial regimen on day 12.

9 Shown are adverse events that occurred in at least $10 \%$ of the patients enrolled in any trial group. Events are listed according to preferred term.

\| Abdominal pain included the following reported terms: abdominal pain, abdominal pain upper, abdominal pain lower, abdominal discomfort, and dyspepsia.

** Liver-related events were reported as liver disorder, elevation in the ALT level, and abnormal liver-function test. The elevation in the ALT level (in the 350-mg dose group) was mild in severity and was considered to be possibly related to the trial regimen. The ALT level increased from a normal baseline value ( $29 \mathrm{IU}$ per liter) to grade 1 at day 29 (51 IU per liter) and at follow-up ( $86 \mathrm{IU}$ per liter). The ALT level was within the normal range at day 65 . The abnormal liver-function test (in the 250-mg dose group) was mild in severity, was considered to be possibly related to the trial regimen, and represented a l-grade shift in the ALT level (grade 2 at baseline to grade 3 from day 7 through the end of the intervention). At follow-up on day 43, the ALT level was near baseline (107 IU per liter); approximately 1 month later, the ALT level had decreased to within the normal range. The liver disorder (in the 350-mg dose group) resulted in discontinuation of the trial regimen (discussed above). 
to $82 \%$ ). Gastrointestinal adverse events were more common at the doses of $250 \mathrm{mg}$ and $350 \mathrm{mg}$ than at lower doses, and a small number of liver abnormalities were observed at the highest doses in patients with extensive previous use of androgens. The side-effect profile in this trial was consistent with a trial involving healthy volunteers, in which gastrointestinal adverse events were more commonly reported in higher BCX7353 dose groups. ${ }^{10}$

The effectiveness of BCX7353 was further supported by secondary efficacy end points involving a post hoc hierarchical analysis, with substantial improvements observed in patients' quality of life at the $125-\mathrm{mg}$ dose level, although post hoc $\mathrm{P}$ values should be interpreted with some caution. The mean improvement (change from baseline) in the AE-QoL total score was greater than 4 times the minimal clinically important difference of 6 points. ${ }^{13}$ In addition, patients who received BXC7353 at doses of $125 \mathrm{mg}$ or more had lower rates of attacks resulting in treatment and a lower overall severity of illness than those who received placebo.

An analysis of attack rates according to dose and exposure in this trial supports a hypothesis that drug exposures that are maintained above a threshold concentration at least 4 times the $\mathrm{EC}_{50}$ correlate with clinically meaningful reductions in attack rates. In this trial, only doses of $125 \mathrm{mg}$ or more provided trough concentrations at least 4.0 times the $\mathrm{EC}_{50}$ with corresponding significant differences in the attack rate as compared with placebo. A $62.5-\mathrm{mg}$ dose provided a mean $\mathrm{C}_{\text {tau }}$ of 1.8 times the $\mathrm{EC}_{50}$, with no significant difference in attack rate as compared with placebo $(\mathrm{P}=0.64)$, whereas a $125 \mathrm{-mg}$ dose provided a mean $\mathrm{C}_{\text {tau }}$ of 5.0 times the $\mathrm{EC}_{50}$, with a corresponding difference in the attack rate of $73.8 \%$ as compared with placebo $(\mathrm{P}<0.001)$.

These data are consistent with exposureresponse results from a phase 3 trial of a subcu- taneous preparation of $\mathrm{C} 1$ inhibitor (Clinical Study for Optimal Management of Preventing Angioedema with Low-Volume Subcutaneous C1Inhibitor Replacement Therapy [COMPACT]). ${ }^{14}$ In that trial, a clinically meaningful reduction in the frequency of attacks correlated with a trough plasma level of $\mathrm{C} 1$ inhibitor equal to 5.3 to 6.3 times the $\mathrm{EC}_{50}$ of $\mathrm{C} 1$ inhibitor on plasma kallikrein, which was determined by the same assay

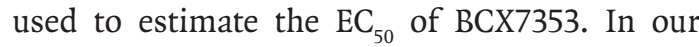
trial, trough concentrations that were achieved at the $250-\mathrm{mg}$ and $350-\mathrm{mg}$ doses were 14 to 20 times the $\mathrm{EC}_{50}$, which suggests that these doses are in excess of those required to achieve clinically meaningful reductions in the attack rate.

In a phase 3 study (OPuS-2) of avoralstat, a first-generation oral kallikrein inhibitor administered three times a day, efficacy in preventing angioedema attacks in patients with hereditary angioedema was not shown. ${ }^{15}$ As compared with BCX7353, avoralstat had inferior pharmacokinetic properties, with low oral bioavailability, a short half-life, and a food effect on plasma exposure. Reported plasma concentrations of avoralstat from the OPuS-2 study were highly variable, with many time points having drug levels below the target therapeutic concentration of 4 times the $\mathrm{EC}_{50}$ for the drug. The results of the OPuS-2 trial further support the hypothesis that maintenance of drug levels above a threshold level for plasma kallikrein suppression is important in reducing the frequency of angioedema attacks.

In conclusion, this trial showed both proof of concept and dose response of orally administered BCX7353 in the prevention of angioedema attacks through short-lasting kallikrein inhibition. Longer studies will need to be performed to assess the safety profile of long-term dosing.

Supported by BioCryst Pharmaceuticals.

Disclosure forms provided by the authors are available with the full text of this article at NEJM.org.

The authors' full names and academic degrees are as follows: Emel Aygören-Pürsün, M.D., Anette Bygum, M.D., D.M.Sci., Vesna Grivcheva-Panovska, M.D., Markus Magerl, M.D., Jochen Graff, M.D., Urs C. Steiner, M.D., Olivier Fain, M.D., Aarnoud Huissoon, M.B., Ph.D., Tamar Kinaciyan, M.D., Henriette Farkas, M.D., Ph.D., D.Sc., Ramon Lleonart, M.D., Hilary J. Longhurst, F.R.C.P., Ph.D., F.R.C.Path., William Rae, M.R.C.P., Massimo Triggiani, M.D., Werner Aberer, M.D., Mauro Cancian, M.D., Ph.D., Andrea Zanichelli, M.D., William B. Smith, M.B., B.S., Ph.D., Maria L. Baeza, M.D., Ph.D., Aurelie Du-Thanh, M.D., Mark Gompels, M.B., B.S., Teresa Gonzalez-Quevedo, M.D., Ph.D., Jens Greve, M.D., Mar Guilarte, M.D., Ph.D., Constance Katelaris, M.D., Ph.D., Sylvia Dobo, M.D., Melanie Cornpropst, Ph.D., Desiree Clemons, M.S., Lei Fang, M.S., Phil Collis, Ph.D., William Sheridan, M.B, B.S., Marcus Maurer, M.D., and Marco Cicardi, M.D. 
The authors' affiliations are as follows: the Division of Hematology, Oncology, and Hemostaseology, Department of Children and Adolescents, Angioedema Center, University Hospital Frankfurt (E.A.-P.), and the Fraunhofer Institute for Molecular Biology and Applied Ecology, Translational Medicine and Pharmacology (J. Graff), Frankfurt, the Department of Dermatology and Allergy, CharitéUniversitätsmedizin Berlin, Berlin (M. Magerl, M. Maurer), and the Department of Otorhinolaryngology and Head and Neck Surgery, Ulm University Medical Center, Ulm (J. Greve) — all in Germany; the Department of Dermatology and Allergy Center, Odense University Hospital, Odense, Denmark (A.B.); Public Health Institution University Clinic of Dermatology, School of Medicine, University Sts. Cyril and Methodius, Skopje, Macedonia (V.G.-P.); the Department of Clinical Immunology, University Hospital Zurich, Zurich, Switzerland (U.C.S.); Assistance Publique-Hôpitaux de Paris Hôpital Saint Antoine, Sorbonne Université, Paris (O.F.), and the Department of Dermatology, Université de Montpellier, Montpellier (A.D.-T.) - both in France; the Allergy and Immunology West Midlands, Birmingham Heartlands Hospital, Birmingham (A.H.), Barts Health NHS Trust-Royal London Hospital, London (H.J.L.), the National Institute for Health Research Southampton Clinical Research Facility, Southampton (W.R.), and the North Bristol NHS Trust, Southmead Hospital, Bristol (M. Gompels) - all in the United Kingdom; the Department of Dermatology, Medical University of Vienna, Vienna (T.K.), and the Department of Dermatology and Venereology, Medical University of Graz, Graz (W.A.) — both in Austria; the Hungarian Angioedema Reference Center, 3rd Department of Internal Medicine, Semmelweis University, Budapest, Hungary (H.F.); the Allergology Unit, Department of Internal Medicine, Hospital Universitario Bellvitge de L'Hospitalet de Llobregat (R.L.) and Hospital Universitari Vall d'Hebron, Vall d'Hebron Research Institute (M. Guilarte), Barcelona, Hospital General Universitario Gregorio Marañón, Biomedical Research Network on Rare Diseases (Centro de Investigación Biomédica en Red de Enfermedades Raras)-Unit 761, Institute for Health Research, Gregorio Marañon, Madrid (M.L.B.), and Hospital Universitario Virgen del Rocío, Seville (T.G.-Q.) - all in Spain; the Division of Allergy and Clinical Immunology, University of Salerno, Salerno (M.T.), the Department of Medicine, University of Padua, Padua (M. Cancian), and Azienda Socio Sanitaria Territoriale Fatebenefratelli Sacco-Università degli Studi di Milano, Milan (A.Z., M. Cicardi) - all in Italy; the Department of Clinical Immunology and Allergy, Royal Adelaide Hospital, Adelaide, SA (W.B.S.), and Campbelltown Hospital, Immunology and Allergy, Western Sydney University, Sydney (C.K.) — both in Australia; and BioCryst Pharmaceuticals (S.D., M. Cornpropst, D.C., P.C., W.S.) and PharStat (L.F.) — both in Durham, NC.

\section{REFERENCES}

1. Zuraw BL. Hereditary angioedema. N Engl J Med 2008;359:1027-36.

2. Longhurst $\mathrm{H}$, Cicardi M. Hereditary angio-oedema. Lancet 2012;379:474-81.

3. Bork K, Hardt J, Witzke G. Fatal laryngeal attacks and mortality in hereditary angioedema due to C1-INH deficiency. J Allergy Clin Immunol 2012;130:692-7. 4. Nordenfelt P, Nilsson M, Lindfors A, Wahlgren CF, Björkander J. Health-related quality of life in relation to disease activity in adults with hereditary angioedema in Sweden. Allergy Asthma Proc 2017;38: 447-55.

5. Pappalardo E, Cicardi M, Duponchel C, et al. Frequent de novo mutations and exon deletions in the C1inhibitor gene of patients with angioedema. J Allergy Clin Immunol 2000;106:1147-54.

6. Patston PA, Gettins P, Beechem J, Schapira M. Mechanism of serpin action: evidence that $\mathrm{C} 1$ inhibitor functions as a suicide substrate. Biochemistry 1991;30: 8876-82.
7. Bork K, Bygum A, Hardt J. Benefits and risks of danazol in hereditary angioedema: a long-term survey of 118 patients. Ann Allergy Asthma Immunol 2008;100:153-61. 8. Cicardi M, Castelli R, Zingale LC, Agostoni A. Side effects of long-term prophylaxis with attenuated androgens in hereditary angioedema: comparison of treated and untreated patients. J Allergy Clin Immunol 1997;99:194-6.

9. Zuraw BL, Davis DK, Castaldo AJ, Christiansen SC. Tolerability and effectiveness of 17- $\alpha$ alkylated androgen therapy for hereditary angioedema: a re-examination. J Allergy Clin Immunol Pract 2016; 4(5):948-955.e15.

10. Cornpropst M, Dobo S, Collier J, et al. $\mathrm{BCX} 7353$, a potent inhibitor of plasma kallikrein, shows sustained maximal enzyme inhibition when dosed orally once daily: results from a phase 1 trial in healthy subjects. J Allergy Clin Immunol 2016;137:AB401. abstract.

11. Weller K, Groffik A, Magerl M, et al.
Development, validation, and initial results of the Angioedema Activity Score. Allergy 2013;68:1185-92.

12. Weller K, Groffik A, Magerl M, et al. Development and construct validation of the Angioedema Quality of Life Questionnaire. Allergy 2012;67:1289-98.

13. Weller K, Magerl M, Peveling-Oberhag A, Martus P, Staubach P, Maurer M. The Angioedema Quality of Life Questionnaire (AE-QoL) - assessment of sensitivity to change and minimal clinically important difference. Allergy 2016;71:1203-9. 14. Longhurst $\mathrm{H}$, Cicardi $M$, Craig T, et al. Prevention of hereditary angioedema attacks with a subcutaneous $\mathrm{C} 1$ inhibitor. N Engl J Med 2017;376:1131-40.

15. Riedl MA, Aygören-Pürsün E, Baker J, et al. Evaluation of avoralstat, an oral kallikrein inhibitor, in a phase 3 hereditary angioedema prophylaxis trial: the OPuS-2 study. Allergy 2018 April 24 (Epub ahead of print).

Copyright (c) 2018 Massachusetts Medical Society.

SPECIALTIES AND TOPICS AT NEJM.ORG

Specialty pages at the Journal's website (NEJM.org) feature articles in cardiology, endocrinology, genetics, infectious disease, nephrology, pediatrics, and many other medical specialties. 